\title{
Umwelt und Entwicklung Beiträge der Privatwirtschaft insbesondere im Bereich der Umwelt- und Raumplanung
}

Peter Gresch

\section{CpenEdition}

\section{Journals}

Electronic version

URL: http://journals.openedition.org/sjep/1383

DOI: $10.4000 /$ sjep. 1383

ISSN: 1663-9677

Publisher

Institut de hautes études internationales et du développement

\section{Printed version}

Date of publication: 1 janvier 1992

Number of pages: 199-204

ISSN: $1660-5926$

\section{Electronic reference}

Peter Gresch, « Umwelt und Entwicklung Beiträge der Privatwirtschaft insbesondere im Bereich der Umwelt- und Raumplanung », Schweizerisches Jahrbuch für Entwicklungspolitik [Online], 11 | 1992, Online erschienen am: 19 Mai 2013, abgerufen am 08 September 2020. URL : http:// journals.openedition.org/sjep/1383; DOI : https://doi.org/10.4000/sjep.1383 


\title{
Umwelt und Entwicklung Beiträge der Privatwirtschaft insbesondere im Bereich der Umwelt- und Raumplanung
}

\author{
Peter Gresch
}

\section{Einleitung}

In diesem Artikel geht es darum, darzustellen, welche Probleme sich im Umweltbereich in den Ländern der Dritten Welt stellen und welche Beiträge Schweizer Ingenieure und Berater zur Lösung dieser Probleme leisten können. Die Ausführungen dienen dem Anliegen dieser Schrift, die bestehenden Aussenbeziehungen unseres Landes sichtbar zu machen und Hinweise auf mögliche weitere Aufgabenfelder zu geben.

\section{Problemstellungen in der Dritten Welt}

Um das Wesentliche nicht aus den Augen zu verlieren, werden als Einstieg einige Passagen aus dem Bericht «Global 2000" zitiert (1):

"Wenn sich die gegenwärtigen Entwicklungstrends fortsetzen, wird die Welt im Jahre 2000 noch überbevölkerter, verschmutzter, ökologisch noch weniger stabil und für Störungen anfälliger sein als die Welt, in der wir heute leben."... «In absoluten Zahlen wird die Bevölkerung im Jahre 2000 schneller zunehmen als heute, nämlich um 100 Millionen Menschen jährlich, im Vergleich zu 75 Millionen im Jahre 1975." (Seiten 25/26).

Diese Bevölkerungsexplosion ist sicher eines der prägenden Merkmale insbesondere der Länder der Dritten Welt. Durch das Bevölkerungswachstum steigt der Platzbedarf und die für Wohnnutzung und Landwirtschaft zur Verfügung stehenden Flächen werden knapper, das heisst, die Konkurrenz um die Nutzung des Bodens und damit die räumlichen Konflikte werden zunehmen. Die (ökonomisch) schwächeren Nutzungen in den Städten müssen den stärkeren Nutzun- 
gen weichen und werden immer weiter vom Zentrum weg an die Peripherie gedrăngt. Dadurch werden die Transportdistanzen grösser, der Energieaufwand wächst und die Emissionen von Luftschad-stoffen und Lärm nehmen zu. Die Lebensqualität sinkt. Ferner muss das Wasser einerseits für immer mehr Menschen ausreichen und wird andererseits in zunehmendem Masse verschmutzt. Um den zunehmenden Bedart an Energie zu decken, werden nicht erneuerbare Ressourcen beansprucht, werden Wälder abgeholzt und Wasserkraftwerke gebaut.

Mit dieser kurzen Charakterisienung der Grundprobleme im Bereich Entwicklung und Umwelt kann es sich sicher nicht um eine umfassende Einführung in den Problemkreis Entwicklung und Umwelt handeln. Vielmehr geht es darum, die Grundlage für die nachfolgenden Ausführungen zu legen.

\section{Die Aussenbeziehungen der Schweiz zur Dritten Welt}

Die ökonomischen Aussenbeziehungen der Schweiz zu Ländern der Dritten Welt bestehen entweder im Handel mit Waren oder im Handel mit Dienstleistungen. Zum Handel mit Waren, über den in diesem Jahrbuch wiedenum ausführlich berichtet wird, gehören gemäss Eidg. Oberzolldirektion folgende Gruppen (2): Rohstoffe und Halbfabrikate; Energieträger; Investitionsgüter und Ausrüstungsgüter; Konsumgüter.

In diesem Artikel wird auf diese Handelsbereiche nicht eingegangen. Vielmehr konzentrieren sich die Ausführungen auf den Dienstleistungsbereich. Insbesondere geht es um die Dienstleistungen im Bereich der Beratungs- und Planungstătigkeit einer grossen Ingenieurunternehmung. Es sind demnach hier nicht der Export von Waren im Vordergrund des Interesses, sondern der Export von naturwissenschaftlichem Wissen, von Methoden, Vorgehensweisen, Verfahren und Denkmustern.

Aufgrund der finanziellen Lage der Länder der Dritten Welt und der grossen Differenz bei den Löhnen ist es praktisch ausgeschlossen, dass Aufträge ohne finanzielle Mitwirkung der öffentlichen Hand - hier aus der Schweiz - erteilt und abgewickelt werden könnten. Mandate aus der Dritten Welt an Berater in der Schweiz basieren weitgehend auf den Mitteln der öffentlichen Entwicklungszusammenarbeit. Bezüglich der Art der Projekte sind es vor allem grosse Infrastrukturvorhaben und grosse Industrieanlagen, die unter Beizug und Federführung von schweizerischen Unternehmungen projektiert werden. Es handelt sich bei diesen Projekten um Kraftwerke, Entsalzungsanlagen, Bahnprojekte, Bewässerungsprojekte usw.

\section{Umweltschutz In der Dritten Welt}

Umweltschutz kann in verschiedenen Formen auftreten:

a) Massnahmen treffen, um verschmutztes Wasser, verschmutzte Böden, verschmutzte Luft zu behandeln (Sanierung von angerichtetem Schaden). 
b) Massnahmen treffen, um die Verschmutzung von Wasser, Böden und Luft zu verhindern (Vorsorge).

Es gibt also einerseits Anlagen, die direkt und unmittelbar dem Schutz der Umwelt dienen (Kläranlagen, Kehrichtverbrennungsanlagen, usw.). Andererseits gibt es Anlagen, die andern Zwecken dienen, aber eine Rolle bezüglich der Belastung bzw. Schonung der Umwelt spielen.

Wir müssen davon ausgehen, dass Kläranlagen und Kehrichtverbrennungsanlagen zwingend notwendige Bestandteile jeder Umweltstrategie sein müssen. Da das Geld für diesen technischen Umweltschutz aber häufig nicht vorhanden sein dürtte, erhălt die Vorsorge, das heisst die Verhinderung von Umweltbelastungen und -schäden höchste Priorität. Wir wollen uns deshalb mit der Vorsorge beim Schutz unserer Umwelt und unseres Lebensraumes befassen.

Aufgrund der Tatsache der Fremdfinanzierung grosser Infrastruktur- und Industrieanlagen werden zunehmend im Zusammenhang mit der Kreditgenehmigung (durch die Weltbank oder die DEH, usw.) neben den Projektplänen auch Berichte verlangt, die Auskunft geben über die zu erwartenden Umweltauswirkungen (Environmental Impact Assessment Studies, EIA oder Umweltverträglichkeitsberichte, UVB). Diese Berichte werden auch in Ländern verlangt, wo noch keine entsprechende Umweltschutzgesetzgebung besteht. In diesen Fällen fehlen häufig die Regelungen über die Beurteilung und Prüfung der Vorhaben im Rahmen der staatlichen Organisation der Empfängerländer. Deshalb ist meisten die geld- bzw. die kreditgebende Instanz die Stelle, die die Berichte in ihren Aussagen prüft und die Ergebnisse im Rahmen der Ent-scheidungsfindung zum Projekt berücksichtigt.

Die Durchführung von Umweltverträglichkeitsabklärungen sind in diesen Ländern vieffach noch Pionierarbeiten. Vorerst muss deshalb die Arbeit noch vollumfănglich von Büros aus den Geldgeberländern geleistet werden. Zunehmend wird es aber so sein, dass der einheimische akademische Nachwuchs mit Teilarbeiten (Inventaren, Dokumentation, usw.) betraut wird, bis schliesslich zu einem bestimmten Zeitpunkt die ausländischen Berater für die Bearbeitung von Standardfällen gar nicht mehr gebraucht werden.

Die Einbeziehung einheimischen Nachwuchses in die Arbeit erfordert vom Berater neben fachlicher Kompetenz auch didaktische Fähigkeiten. Aus diesem Grunde ist es begrüssenswert und nützlich, wenn unsere Berater und Mitarbeiter nebenamtlich auch in der Ausbildung an Hoch- und Ingenieurschulen oder Universităten engagiert sind.

Gewissermassen im "Windschatten" von Aufträgen für die Projektienung von Infrastruktur- und Industrieanlagen wird so eine Form von Entwicklungszusammenarbeit geleistet, die immer mehr Bedeutung bekommt, weil es sich bei der Ausbildung von Menschen wohl um die nachhaltigste Art von Förderung handelt.

Die Erstellung eines Berichtes über die zu enwartenden Umweltauswirkungen zu einem Projekt erst zum Zeitpunkt, wo der Standort bereits festgelegt ist, kann aber letztlich nicht befriedigen. Die Beschreibung und Beurteilung der Emissionen, Immissionen, usw. beziehen sich dann nur noch auf den einen konkreten 
Standort. Es zeichnet sich aber immer deutlicher ab, dass bereits für die Evaluation der verschiedenen Standorte Oekologen, Raumplaner und Geographen für die Beschreibung und Beurteilung beizuziehen sind, denn auf dieser Stufe ist ein systematischer Vergleich bezüglich der Vor- und Nachteile der verschiedenen Lösungen und dadurch eine Verhinderung grober Fehler noch möglich.

Mit diesem Schritt der Variantenevaluation verlassen wir aber den Geltungsbereich der Umweltschutzgesetzgebung (Umweltverträglichkeitsprüfung) und kommen, jedenfalls in der Schweiz, in den Geltungsbereich der Raumplanungsgesetzgebung. Nach Maurer (3) ist Raumplanung ein Mittel zur Fördenung der allgemeinen Wohlfahrt. Die Aufgabe der Raumplanung besteht in der Steuerung jener Vorgänge, welche zukünftige räumliche Ordnung und damit Umweltqualität massgebend beeinflussen. Die Standortentscheide für grosse Infrastruktur- und Industrievorhaben sind in diesem Sinne sicher derartige Vorgänge.

In der neueren Raumplanung wird zwischen Richtplanung und Nutzungsplanung unterschieden. Die Nutzungsplanung regelt allgemein verbindlich die zulässige Nutzung des Bodens, während die Richtplanung der Koordination raumwirksamer Tätigkeiten dient. Bei der Richtplanung besteht der erste Schritt der Koordination darin, dass Absichten über künftige Vorhaben angemeldet werden müssen. Die Anmeldung von Absichten für die Realisierung von grossen, raumwirksamen Vorhaben will sicherstellen, dass alle an der Nutzung eines Gebietes Interessierten von den Absichten Anderer Kenntnis bekommen und die allfällig daraus resultierenden Konflikte sichtbar gemacht werden können. Mit dem Sichtbarmachen sollen Vor- und Nachteile enwogen und ungünstige oder unerwünschte Ent-wicklungen rechtzeitig gestoppt werden.

Dieses in der Schweiz im Rahmen der kantonalen Richtplanung vorgeschriebene Prozedere liesse sich auch im Ausland für die Evaluation verschiedener Standorte anwenden. Genauso wie bei der Beschreibung und Beurteilung der zu erwartenden Auswirkungen eines konkreten Vorhabens an einem konkreten Standort ist es erforderlich, die vorangehende Standortevaluation ebenfalls mit einem Team von qualifizierten Umweltfachleuten durchzuführen. Die Aufgabe ist jedoch wegen des Problems des Vergleichen-müssens noch um eine Stufe anspruchsvoller. Aber auch in diesen Aufgabenbereich lassen sich mit der Zeit die einheimischen Kaderleute einarbeiten.

Oberhalb der Projekt- und der Standortevaluationsebene drăngt sich aufgrund der gemachten Erfahrungen nun noch eine dritte Beratungsebene auf: Die Ebene der Planung der künftigen Nutzung des Bodens für ein grösseres zusammenhängendes Gebiet. Die qualifizierte Behandlung dieser Ebene und die Bereitstellung verbindlicher Vorgaben über die Nutzung des Bodens ist Voraussetzung, um eine Standortevaluation zweckmässig und korrekt durchführen zu können. Die Prüfung der Verträglichkeit der verschiedenen Lösungen bezüglich der raumplanerischen Vorgaben ist notwendig, um nicht über geltende rechtliche Vorschriften zu stolpem.

Auch auf dieser Ebene ist eine Arbeitsteilung zwischen den Beratern aus der Schweiz und den einheimischen Fachkräften möglich: Das Beraterteam hifft bei der Formulierung der enwünschten räumlichen Entwicklung und bei der Herausar- 
beitung der hauptsächlichsten räumlichen und umweltmăssigen Probleme und Konflikte, die einer Lösung harren. Im Rahmen der Beurteilung der Lage macht das Beraterteam Vorschläge für die Formulierung von Auftrăgen zur Bearbeitung der einzelnen Probleme und Konflikte. In diesen Aufträgen wird formuliert, wer, mit wem, bis wann über was zu verhandeln und eine Lösung des Konfliktes oder Problems zu finden hat. Die Bearbeitung dieser einzelnen Koordinationsaufgaben kann dann wieder unter Begleitung durch die externen Berater durchaus von den einheimischen Fachkrätten übernommen werden. (Entsprechende Modelle sind auch in der Schweiz in der Zusammenarbeit zwischen privatwirtschaftlichen Beratungsteams und kantonalen Raumplanungsstellen etabliert.)

\section{Die Bedeutung einer effizienten Raum- und Umweltplanung}

Im Vordergrund der Anstrengungen in der Dritten Welt muss sicher die Kontrolle und Reduktion des Bevölkerungswachstums liegen. Auf der andern Seite zeigt das Beispiel der entwickelten Länder, dass mit einer sorgsamen und zweckmässigen Nutzung des Bodens und mit einer umweltverträglichen Realisierung grosser Anlagen die Umwelt- und Lebensqualität auch in den Ländern der Dritten Welt nicht unbedingt sinken muss, bzw. eine wesentlich grössere Zahl an Menschen ernährt und beschäftigt werden könnte, ohne dass die Umwelt nachhaltigen Schaden erleiden würde.

Die Entwicklung der Umweltsituation in den Ländern der Dritten Welt kann uns insofern nicht gleichgültig sein, da sich in diesen Gebieten, wie man heute vermutet (4), die für die Aufrechterhaltung der global gesehen optimalen Lebensbedingungen wichtigen Land- und Wasserflächen befinden (Schilfregionen, Feuchtgebiete, Regenwälder, usw.). Die Zerstörung der Biosphäre in diesen Gebieten würde sich, fürchten Fachleute, ungünstig, ja verheerend auf die Selbstregulierungskraft der Erde (GAIA) auswirken. Somit ist es wohl gut gemeint, aber völlig unzureichend, nur bei einzelnen Projekten mit UVP-Studien für die Erhaltung einer intakten Umwelt sorgen zu wollen. Auf allen drei Stufen sind Arbeiten nötig: Auf der Ebene der verbindlichen Regelung der Nutzung des Bodens in rechtskräftigen Nutzungs- und Richtplänen, auf der Ebene der Evaluation von verschiedenen Standorten für ein Vorhaben und auf der Stufe der Beschreibung der Auswirkungen eines konkreten Vorhabens.

\section{Beispiel Vietnam Yali Falls}

Beim vorliegenden Beispiel handelt es sich um das Projekt zum Bau eines Wasserkraftwerkes an einem Seitenfluss des Mekong Rivers in der Volksrepublik Vietnam. Im Anschluss an die Prüfung von verschiedenen Möglichkeiten zur Wasserkraftnutzung an obigem Flusssystem wurde von Japanern eine Machbarkeitsstudie für ein Kraftwerk bei den Yali Falls durchgeführt. Im Frühjahr 1991 schrieb das Sekretariat des Mekong Komitees in Bangkok die Arbeiten für einen Umweltverträglichkeitsbericht und einen Bericht über die Finanzierung des Kraftwerkes aus. Das Mekong Komitee ist eine Organisation der UNO, wobei un- 
ter anderem auch die Schweiz Beitrăge leistet. Das Yali Projekt, bzw. die ausgeschriebene Studie wird schwergewichtig durch die Schweiz, d.h. durch die DEH finanziert. Dank dieser Finanzierung ist es möglich, das Berater-Potential einer schweizerischen Ingenieurunternehmung (bzw. ihrer Oekologen) einzusetzen und zu nutzen. Da die Arbeiten von der Schweiz bezahlt werden, gelten, obwohl das Projekt in einem andern Land (noch ohne entsprechende Umweltschutzgesetzgebung) liegt, die gleichen Vorschriften bezüglich der UVP, wie wenn die Anlage in der Schweiz gebaut würde.

Es ist vorgesehen, insgesamt 10 bis 15 Vietnamesen in die Untersuchungen zu integrieren, um den Ausbildungseffekt, wie oben beschrieben, zu erreichen. Zudem drängt sich im Falle des Yali Projektes die Behandlung raumplanerischer Fragen auf: Die Umlegung von Verkehrswegen, die Neuanlage von Siedlungen und Dörfern, die Reorganisation der Landnutzung, usw.

\section{Zusammenfassung}

In diesem Beitrag ging es danum, aus der Palette der Beiträge der Schweiz in der Entwicklungszusammenarbeit speziell auf die privatwirtschaftlichen Dienstleistungen im Bereich der Raumplanung und Umweltberatung einzugehen. Obwohl mit den Berichten über die Umweltauswirkungen der einzelnen Projekte ein wesentlicher Schritt in Richtung eines sorgsamen Umgangs mit unserer Umwelt und der Natur gemacht wird, genügt die einzelfallweise Behandlung raum- und umweltrelevanter Fragen nicht. Die Beratertätigkeit ist auf die Verfahren zur Koordination raumwirksamer Tätigkeiten auszudehnen, wobei uns von der Schweiz aus die eigenen Erfahrungen im Zusammenhang mit der kantonalen Richtplanung und den Arbeiten an UVP's zugute kommen.

\section{Anmerkungen}

1 Council on Environmental Quality und Aussenministerium, "Global 2000 - Der Bericht an den Präsidenten", Deutschsprachige Ausgabe, Verlag Zweitausendundeins, Frankfurt am Main, 1981.

2 Institut Universitaire d'Etudes du Developpement, «Jahrbuch Schweiz - Dritte Welt 1991×, Genf, 1991.

3 Maurer J., "Grundzüge einer Methodik der Raumplanung», Schriftenreihe Nr. 14, Institut für Orts-, Regional- und Landesplanung ETHZ, Zürich, 1973.

4 Lovelock J.E., "GAIA - a new look at life on earth», Oxford University Press, 1987. 